\title{
MIMO Radar Waveform Design with Prior Information of Targets in the Presence of Signal-Dependent Noise
}

\author{
HongFeng Wang \\ School of Computer Science and Technology, Zhoukou Normal University, \\ Zhoukou, 466001, China \\ whfwlj202868@163.com
}

\begin{abstract}
Multiple-input multiple-output (MIMO) radar waveforms design with specified properties has a number of superiority over its phased-array counterpart, such as clutter suppression and interference mitigation. In this paper, we consider the problem of waveform optimization with prior information on targets of interest to improve the parameter estimation performance of MIMO radar in the presence of signal-dependent noise, which is based on the constrained Cramer-Rao bound (CRB). The waveform covariance matrix (WCM) is designed to minimize the trace of the constrained CRB such that the parameter estimation performance can be improved. In order to solve the resultant nonlinear optimization problem, a novel diagonal loading (DL) based method is proposed to relax this optimization issue as a semidefinite programming (SDP) one, which can be solved very efficiently. Following that, an optimal solution to the initial issue can be obtained via the least squares (LS) fitting of the solution acquired by the relaxed one. The effectiveness of the proposed method is verified by numerical examples, as compared to the uncorrelated waveforms.
\end{abstract}

Keywords: Multi-input multi-output (MIMO) radar, waveform design, diagonal loading (DL), signal-dependent noise, constrained Cramer-Rao bound (CRB), semidefinite programming (SDP)

\section{Introduction}

Multi-input multi-output (MIMO) radar has been intensively researched recently [120]. MIMO radar is defined as a radar system with multiple antennas to simultaneously transmit arbitrary waveforms other than coherent waveforms in traditional phased-array radars, which is the so-called waveform diversity [1]. In terms of the spacing between its antennas, MIMO radar can be classified into two categories shown as: (1) MIMO radar with widely separated antennas (e.g., [2]), and (2) MIMO radar with colocated antennas (e.g., [1]). The former employs the widely-spaced transmitting and receiving elements along with diverse transmitted waveforms to view the different aspects of the target thereby improving the target detection performance. In contrast, the latter employs the close-spaced elements in transmitting and receiving arrays to obtain the identical target radar cross sections (RCSs) observed from all transmit/receive paths, which can utilize the waveform diversity to increase the virtual aperture of the receiving array [1]. Accordingly, it has several advantages including improved parameter identifiability [3-4], and more flexibility for transmit beampattern design [5-20]. In this paper, we consider MIMO radar with collocated antennas.

For both types of MIMO radar, a particularly critical issue is the waveform optimization [5-20], which has received considerable attention recently. According to the objects needed to be optimized, the current design methods can be divided into two categories: (1) only the transmitter to be designed [5-11], and (2) the transmitter and receiver to be designed jointly [12-20]. Specific issues that have been considered in first 
category include the transmit beam pattern design and radar ambiguity function design. In [5-6], the waveform covariance matrix (WCM) was designed to attain a desired beam pattern, while the constant modulus signal design is considered in [7-9]. The robust waveform covariance matrix is considered in [10] with a general class of cost functions encompassing several known performance measures, e.g., signal-to-disturbance ratio. Meanwhile, the spatial-range-Dopper domain characteristics of the transmitted waveforms were considered jointly in [11] to improve the radar performance. Some works have been done in the second category approaches to investigate the waveform design problem by jointly optimizing the radar transmitter and receiver. In [12], the transmit waveforms were optimized for multiple point targets based on several design criteria, for example, minimizing the trace of the Cramér-Rao Bound (CRB) matrix. The output signal-tointerference-plus-noise ratio (SINR) was maximized in [13] to improve the detection performance for MIMO radar in the case of extended target by exploiting a gradient based method. Unfortunately, the method proposed in [13] cannot guarantee nondecreasing SINR in each iteration step. In order to guarantee convergence, a new iterative algorithm was derived in [14].The mutual information between the received waveforms and the target radar signatures was employed to design the transmit waveform for extended target [15-17]. Other information theoretic based transmit waveform designs were considered in [18] for multiple extended targets. In [19], MIMO waveform was devised by minimizing the estimation error of the minimum mean squared error (MMSE) estimators for uncorrelated and correlated targets. The joint optimization of the WCM and receiver is investigated to improve the parameter estimation performance in [20].

In [12], J. Li et. al., has investigated the problem of waveform design for parameter estimation of point targets based on CRB in the absence of signal-dependent noise (i.e., clutter). However, it can be seen that the received signal is generally contaminated by clutter in many applications (see, e.g., [8-19]). Moreover, it is well-known that the CRB provides a lower bound on the variance when any unbiased estimator is used without employing any prior information. In fact, in many array signal processing fields, some prior information may be available (see, e.g., [21]), which can be regarded as a constraint on the estimated parameter space. A variant of the CRB for this kind of the constrained estimation problem was developed in [22], which is called the constrained CRB. Based on the discussion above, from the parameter estimation point of view, it is worth considering the waveform optimization problem with prior information in the presence of clutter.

In this paper, we investigate the problem of MIMO radar waveform optimization with prior information of targets of interest in the presence of clutter, which is based on the constrained CRB. The WCM is optimized to minimize the trace of the constrained CRB such that the parameter estimation performance of MIMO radar in the presence of clutter can be improved. In order to solve the resultant complicated nonlinear optimization problem, a novel diagonal loading (DL) [23] based method is proposed to relax this issue as a semidefinite programming (SDP) [24] one, which can be solved very efficiently (may use many methods, see, e.g., [25]). Following that, an optimal solution to the initial optimization problem is constructed through a suitable approximation to an optimal solution of the relaxed one (in a least squares (LS) sense).

The rest of this paper is organized as follows. Section 2 introduces the MIMO radar model, gives the derivation of the CRB, and formulates the waveform optimization problem. Section 3 proposes an DL-based algorithm to solve the nonlinear waveform optimization problem and constructs an optimal solution to the initial issue. Section 4 shows the effectiveness of the proposed method via numerical examples. Finally, Section 5 concludes this paper.

Throughout the paper, matrices and vectors are denoted by boldface uppercase and lowercase letters, respectively. We use $\{\cdot\}^{T},\{\cdot\}^{*}$, and $\{\cdot\}^{H}$ to denote the transpose, conjugate, and conjugate transpose, respectively. $\operatorname{vec}\{\cdot\}$ is the vectorization operator 
stacking the columns of a matrix on top of each other, I denotes the identity matrix, * and $\otimes$ indicates the Khatri-Rao and Kronecker product, respectively. Denote by $\operatorname{tr}\{\cdot\}$, $\operatorname{Re}\{\cdot\}$, and $\operatorname{Im}\{\cdot\}$ the trace, real and imaginary part of a matrix, respectively. The notation $E\{\cdot\}$ stands for the expectation operator, $\operatorname{diag}\{\mathbf{a}\}$ for a diagonal matrix with its diagonal given by the vector a , and $\|\mathbf{A}\|_{F}$ for the Frobenius norm of the matrix $\mathbf{A}$. Given a vector function $\mathbf{f}: \square^{n} \rightarrow \square^{k}$, we denote by $\frac{\partial \mathbf{f}}{\partial \boldsymbol{\theta}}$ the $k \times n$ matrix whose ijth element is $\frac{\partial \mathbf{f}_{i}}{\partial \boldsymbol{\theta}_{j}}$. Finally, the notation $\mathbf{A} \circ \mathbf{B}$ means that $\mathbf{B}-\mathbf{A}$ is positive semidefinite.

\section{Problem Formulation}

Consider a MIMO radar system with $M_{t}$, transmitting elements and $M_{r}$ receiving elements. Let $\Phi=\left[\phi_{1}, \phi_{2}, \cdots, \phi_{M}\right]^{T} \in \square^{M, \times L}$ be the transmitted waveform matrix, where $\phi_{i} \in \square^{L \times 1}, i=1,2, \cdots, M$, denotes the discrete-time baseband signal of the ith transmit element with $L$ being the number of snapshots. Under the assumption that the transmitted signals are narrowband and the propagation is non-dispersive, the received signals by MIMO radar can be expressed as:

$\mathbf{Y}=\sum_{k=1}^{K} \mathbf{a}\left(\theta_{k}\right) \mathbf{v}^{T}\left(\theta_{k}\right) \Phi+\int_{-\pi}^{\pi} \rho(\theta) \mathbf{a}_{c}(\theta) \mathbf{v}_{c}^{T}(\theta) \Phi \mathrm{d} \theta+\mathbf{W}$

where the columns of $\mathbf{Y} \in \square^{M_{r} \times L}$ are the collected data, $\left\{\beta_{k}\right\}_{k=1}^{K}$ are the complex amplitudes proportional to the RCSs of targets with $K$ being the number of targets at the considered range bin, and $\left\{\theta_{k}\right\}_{k=1}^{K}$ denote the locations of these targets. The parameters $\left\{\beta_{k}\right\}_{k=1}^{K}$ and $\left\{\theta_{k}\right\}_{k=1}^{K}$ need to be estimated from $\mathbf{Y}$. The second term in the right hand of the equation above indicates the clutter data collected by the receiver. $\rho(\theta)$ is the reflect coefficient of the clutter patch at $\theta$. The term $\mathbf{w}$ denotes the interference plus noise, which is independent of the clutter. Similar to [12], the columns of $\mathbf{w}$ can be assumed to be independent and identically distributed circularly symmetric complex Gaussian random vectors with mean zero and an unknown covariance $\mathbf{B} \cdot \mathbf{a}\left(\theta_{k}\right)$ and $\mathbf{v}\left(\theta_{k}\right)$ denote, respectively, the receiving and transmitting steering vectors for the target located at $\theta_{k}$, which can be expressed as

$$
\begin{aligned}
& \mathbf{a}\left(\theta_{k}\right)=\left[e^{j 2 \pi f_{0} \tau_{1}\left(\theta_{k}\right)}, e^{j 2 \pi f_{0} \tau_{2}\left(\theta_{k}\right)}, \cdots, e^{j 2 \pi f_{0} \tau_{M_{r}}\left(\theta_{k}\right)}\right]^{T} \\
& \mathbf{v}\left(\theta_{k}\right)=\left[e^{j 2 \pi f_{0} \tilde{\varepsilon}_{1}\left(\theta_{k}\right)}, e^{j 2 \pi f_{0} \tilde{z}_{2}\left(\theta_{k}\right)}, \cdots, e^{j 2 \pi f_{0} \tilde{\mu}_{M_{k}}\left(\theta_{k}\right)}\right]^{T}
\end{aligned}
$$

where $f_{0}$ represents the carrier frequency, $\tau_{m}\left(\theta_{k}\right), m=1,2, \cdots M_{r}$ is the propagation time from the target located at $\theta_{k}$ to the mth receiving element, and $\tilde{\tau}_{n}\left(\theta_{k}\right), n=1,2, \cdots M$, is the propagation time from the nth transmitting element to the target. Also, $\mathbf{a}_{c}(\theta)$ and $\mathbf{v}_{c}(\theta)$ denote the receiving and transmitting steering vectors for the clutter patch at $\theta$, respectively.

If the isorange ring is divided in the cross-range dimension into $N_{C}\left(N_{C} \square N M L\right)$ clutter patches, then (1) can be recast as

$\mathbf{Y}=\sum_{k=1}^{K} \beta_{k} \mathbf{a}\left(\theta_{k}\right) \mathbf{v}^{T}\left(\theta_{k}\right) \Phi+\sum_{i=1}^{N_{c}} \rho\left(\theta_{i}\right) \mathbf{a}_{c}\left(\theta_{i}\right) \mathbf{v}_{c}^{T}\left(\theta_{i}\right) \Phi+\mathbf{W}$ 
where $\mathbf{a}_{c}\left(\theta_{i}\right)$ and $\mathbf{v}_{c}\left(\theta_{i}\right)$ denote the receiving and transmitting steering vectors for the clutter patch at $\theta_{i}$, respectively.

Let $\mathbf{H}_{c}=\sum_{i=1}^{N} \rho\left(\theta_{i}\right) \mathbf{a}_{c}\left(\theta_{i}\right) \mathbf{v}_{c}^{T}\left(\theta_{i}\right)$, according to [26], $\operatorname{vec}\left(\mathbf{H}_{c}\right)$ can be considered as an identically distributed complex Gaussian random vector with mean zero and covariance

$$
\mathbf{R}_{\mathbf{H}_{c}}=E\left[\operatorname{vec}\left(\mathbf{H}_{c}\right) \operatorname{vec}^{H}\left(\mathbf{H}_{c}\right)\right] \pm \mathbf{0}
$$

We now consider the constrained $\mathrm{CRB}$ of the unknown target parameters $\mathbf{x}=\left[\boldsymbol{\theta}^{T}, \boldsymbol{\beta}_{R}^{T}, \boldsymbol{\beta}_{I}^{T}\right]^{T}, \quad$ where $\boldsymbol{\beta}_{R}=\left[\beta_{R, 1}, \beta_{R, 2}, \cdots, \beta_{R, K}\right]^{T}, \quad \boldsymbol{\beta}_{I}=\left[\beta_{I, 1}, \beta_{I, 2}, \cdots, \beta_{I, K}\right]^{T}$, $\boldsymbol{\theta}=\left[\theta_{1}, \theta_{2}, \cdots, \theta_{K}\right]^{T}, \beta_{R}=\operatorname{Re}(\beta)$, and $\beta_{I}=\operatorname{Im}(\beta)$. According to [22], the constrained $\mathrm{CRB}$ can be written as

$\mathbf{J}=\mathbf{U}\left(\mathbf{U}^{H} \mathbf{F} \mathbf{U}\right)^{-1} \mathbf{U}^{H}$

where $\mathbf{F}$ denotes the Fisher information matrix (FIM), and $\mathbf{U}$ satisfies:

$\mathbf{G}(\mathbf{x}) \mathbf{U}(\mathbf{x})=\mathbf{0}, \quad \mathbf{U}^{H}(\mathbf{x}) \mathbf{U}(\mathbf{x})=\mathbf{I}$

in which $G(\mathbf{x})=\frac{\partial \mathbf{g}(\mathbf{x})}{\partial \mathbf{x}}$ is assumed to have full row rank with $\mathbf{g}(\mathbf{x})$ being the equality constraint set on $\mathbf{x}$ and $\mathbf{U}$ is the tangent hyperplane of $\mathbf{g}(\mathbf{x})$ [22].

Following [21] and [22], some prior information of signal or targets of interest can be available in array signal processing, for example, constant modulus constraint on the transmitted waveform, etc.. Due to the fact that the knowledge of one target of interest can be acquired roughly by many methods (see, e.g., [27] for more details), in this paper, the complex amplitude matrix $\beta=\operatorname{diag}\left(\beta_{1}, \beta_{2}, \cdots, \beta_{K}\right)$ can be assumed to be known as:

$$
\begin{aligned}
& g_{i}(\mathbf{x})=\beta_{R, i}-1=0, \quad i=1, \cdots, K \\
& g_{j}(\mathbf{x})=\beta_{I, j}-1=0, \quad j=K+1, \cdots, 2 K
\end{aligned}
$$

Following (7), we can obtain $\mathbf{G}=\left[\mathbf{0}_{2 K \times K}, \mathbf{I}_{2 K \times 2 K}\right]$, where $\mathbf{0}_{2 K \times K}$ denotes a zero matrix of size $2 K \times K$. Hence, the corresponding null space $\mathbf{U}$ can be expressed as

$\mathbf{U}=\left[\begin{array}{ll}\mathbf{I}_{K \times K} & \mathbf{0}_{K \times 2 K}\end{array}\right]^{H}$

Based on the discussion above, the FIM $\mathbf{F}$ with respect to $\mathbf{x}$ can be derived in Appendix A and given by

$$
\mathbf{F}=2\left[\begin{array}{ccc}
\operatorname{Re}\left(\mathbf{F}_{11}\right) & \operatorname{Re}\left(\mathbf{F}_{12}\right) & -\operatorname{Im}\left(\mathbf{F}_{12}\right) \\
\operatorname{Re}^{T}\left(\mathbf{F}_{12}\right) & \operatorname{Re}\left(\mathbf{F}_{22}\right) & -\operatorname{Im}\left(\mathbf{F}_{22}\right) \\
-\operatorname{Im}^{T}\left(\mathbf{F}_{12}\right) & -\operatorname{Im}^{T}\left(\mathbf{F}_{22}\right) & \operatorname{Re}\left(\mathbf{F}_{22}\right)
\end{array}\right]
$$

where,

$$
\begin{aligned}
& \mathbf{F}_{11}=\left\{\left((\dot{\mathbf{V}} * \mathbf{A})^{H}\left(\mathbf{I}+\left(\mathbf{R}_{\Phi} \otimes \mathbf{B}^{-1}\right) \mathbf{R}_{\mathbf{H}_{c}}\right)^{-1}\left(\mathbf{R}_{\Phi} \otimes \mathbf{B}^{-1}\right)(\dot{\mathbf{V}} * \mathbf{A})\right)\right. \\
& +\left((\dot{\mathbf{V}} * \mathbf{A})^{H}\left(\mathbf{I}+\left(\mathbf{R}_{\Phi} \otimes \mathbf{B}^{-1}\right) \mathbf{R}_{\mathbf{H}_{c}}\right)^{-1}\left(\mathbf{R}_{\Phi} \otimes \mathbf{B}^{-1}\right)(\mathbf{V} * \dot{\mathbf{A}})\right) \\
& +\left((\mathbf{V} * \dot{\mathbf{A}})^{H}\left(\mathbf{I}+\left(\mathbf{R}_{\Phi} \otimes \mathbf{B}^{-1}\right) \mathbf{R}_{\mathbf{H}_{c}}\right)^{-1}\left(\mathbf{R}_{\Phi} \otimes \mathbf{B}^{-1}\right)(\dot{\mathbf{V}} * \mathbf{A})\right) \\
& \left.+\left((\mathbf{V} * \dot{\mathbf{A}})^{H}\left(\mathbf{I}+\left(\mathbf{R}_{\Phi} \otimes \mathbf{B}^{-1}\right) \mathbf{R}_{\mathbf{H}_{c}}\right)^{-1}\left(\mathbf{R}_{\Phi} \otimes \mathbf{B}^{-1}\right)(\mathbf{V} * \dot{\mathbf{A}})\right)\right\} \quad\left(\boldsymbol{\beta}^{*} \boldsymbol{\beta}^{T}\right)
\end{aligned}
$$




$$
\begin{aligned}
& \mathbf{F}_{12}=\operatorname{diag}\left(\boldsymbol{\beta}^{*}\right)\left\{\left((\dot{\mathbf{V}} * \mathbf{A})^{H}\left(\mathbf{I}+\left(\mathbf{R}_{\Phi} \otimes \mathbf{B}^{-1}\right) \mathbf{R}_{\mathbf{H}_{c}}\right)^{-1}\left(\mathbf{R}_{\Phi} \otimes \mathbf{B}^{-1}\right)(\mathbf{V} * \mathbf{A})\right)\right. \\
& \left.+\left((\mathbf{V} * \dot{\mathbf{A}})^{H}\left(\mathbf{I}+\left(\mathbf{R}_{\Phi} \otimes \mathbf{B}^{-1}\right) \mathbf{R}_{\mathbf{H}_{c}}\right)^{-1}\left(\mathbf{R}_{\Phi} \otimes \mathbf{B}^{-1}\right)(\mathbf{V} * \mathbf{A})\right)\right\} \\
& \mathbf{F}_{22}=(\mathbf{V} * \mathbf{A})^{H}\left(\mathbf{I}+\left(\mathbf{R}_{\Phi} \otimes \mathbf{B}^{-1}\right) \mathbf{R}_{\mathbf{H}_{c}}\right)^{-1}\left(\mathbf{R}_{\Phi} \otimes \mathbf{B}^{-1}\right)(\mathbf{V} * \mathbf{A}) \\
& \mathbf{R}_{\Phi}=\Phi^{*} \boldsymbol{\Phi}^{T}, \boldsymbol{\beta}=\left[\begin{array}{llll}
\beta_{1}, & \beta_{2}, & \cdots & \beta_{K}
\end{array}\right]^{T}
\end{aligned}
$$

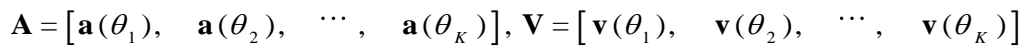

$$
\begin{aligned}
& \dot{\mathbf{A}}=\left[\begin{array}{lll}
\frac{\partial \mathbf{a}\left(\theta_{1}\right)}{\partial \theta_{1}} & \cdots & \frac{\partial \mathbf{a}\left(\theta_{K}\right)}{\partial \theta_{K}}
\end{array}\right], \dot{\mathbf{V}}=\left[\begin{array}{lll}
\frac{\partial \mathbf{v}\left(\theta_{1}\right)}{\partial \theta_{1}} & \cdots & \frac{\partial \mathbf{v}\left(\theta_{K}\right)}{\partial \theta_{K}}
\end{array}\right]
\end{aligned}
$$

The MIMO radar waveform design with prior information of targets of interests to improve the parameter estimation performance in the presence of clutter can now be briefly stated as follows: Optimize the WCM to minimize the constrained CRB under the constraints about the WCM. Based on (5), (8) and (9), under the Trace-opt criterion [12], this optimization problem can be illustrated as:

$$
\begin{array}{ll}
\underset{\mathbf{R}_{\Phi}}{\min } & \operatorname{tr}(\mathbf{J}) \\
\text { s.t. } & \mathbf{R}_{\Phi} \pm \mathbf{0} \\
& \operatorname{tr}\left(\mathbf{R}_{\Phi}\right)=P
\end{array}
$$

where $P$ denotes the total transmitted power. The second constraint holds due to the power transmitted by any transmitting element is more than or equal to zero in practice.

It is obvious that the trace of the CRB matrix, i.e., the objective in (16), is a rather complicated nonlinear function of $\mathbf{R}_{\Phi}$. Moreover, due to the fact that $\mathbf{B} \succ \mathbf{0}, \mathbf{R}_{\Phi} \pm \mathbf{0}, \mathbf{R}_{\mathbf{H}_{c}} \pm \mathbf{0}$, then $\left(\mathbf{R}_{\Phi} \otimes \mathbf{B}^{-1}\right) \mathbf{R}_{\mathbf{H}_{c}}$ is indefinite matrix [29]. Consequently, the problem is difficult to be solved by using the tradition method, for example, the convex optimization method [24].

\section{Proposed DL-Based Method}

In this section, we demonstrate how to obtain an optimal solution of the nonlinear optimization problem in (16). For this purpose, the DL approach, which has been commonly exploited in the robust beamforming (see, e.g., [23]), is employed to $\mathbf{R}_{\Phi}$, and $\mathbf{R}_{\mathbf{H}_{c}}$, respectively, such that

$\tilde{\mathbf{R}}_{\Phi}=\mathbf{R}_{\Phi}+\varepsilon \mathbf{I} \succ \mathbf{0}, \tilde{\mathbf{R}}_{\mathbf{H}_{c}}=\mathbf{R}_{\mathbf{H}_{c}}+\mu \mathbf{I} \succ \mathbf{0}$

where $\varepsilon \square \lambda_{\text {max }}\left(\mathbf{R}_{\Phi}\right), \quad \mu \square \lambda_{\text {max }}\left(\mathbf{R}_{\mathbf{H}_{c}}\right)$ are the so-called loading factors, and $\lambda_{\max }(\cdot)$ denotes the largest eigenvalue of a matrix. In the following, we choose $\varepsilon=\lambda_{\max }\left(\mathbf{R}_{\Phi}\right) / 1000, \mu=\lambda_{\max }\left(\mathbf{R}_{\mathbf{H}_{c}}\right) / 1000$ by numerical examples. By replacing $\mathbf{R}_{\Phi}, \mathbf{R}_{\mathbf{H}_{c}} \quad$ in (9) with $\tilde{\mathbf{R}}_{\Phi}, \tilde{\mathbf{R}}_{\mathbf{H}_{c}}$, respectively, we can obtain $\left(\mathbf{I}+\left(\tilde{\mathbf{R}}_{\Phi} \otimes \mathbf{B}^{-1}\right) \tilde{\mathbf{R}}_{\mathbf{H}_{c}}\right)^{-1}\left(\tilde{\mathbf{R}}_{\Phi} \otimes \mathbf{B}^{-1}\right) \succ \mathbf{0}$.

The proposition below can reformulate the nonlinear objective in (16) with the variable $\mathbf{R}_{\Phi}$ as a linear one, and gives the corresponding linear matrix inequality (LMI) formulations of constraints, which is proved in Appendix B. 
Proposition: Using matrix manipulations, the constraints in (16) can be converted into the following LMIs

$$
\alpha \mathbf{I} \circ \tilde{\mathbf{R}}_{\mathbf{H}_{c}}-\tilde{\mathbf{R}}_{\mathbf{H}_{c}} \mathbf{E} \tilde{\mathbf{R}}_{\mathbf{H}_{c}} \circ \beta \mathbf{I}
$$

where

$$
\mathbf{E}=\left(\mathbf{I}+\left(\tilde{\mathbf{R}}_{\Phi} \otimes \mathbf{B}^{-1}\right) \tilde{\mathbf{R}}_{\mathbf{H}_{c}}\right)^{-1}\left(\tilde{\mathbf{R}}_{\Phi} \otimes \mathbf{B}^{-1}\right)
$$

and $\alpha, \beta$ are given in (51) and (52), respectively. With this Proposition, the problem in (16) can be cast as SDP relying on the following lemma [29, pp.472]:

Lemma 1 (Schur's Complement): Let $\mathbf{Z}=\left[\begin{array}{cc}\mathbf{A} & \mathbf{B}^{H} \\ \mathbf{B} & \mathbf{C}\end{array}\right]$ be a Hermitian matrix with $\mathbf{C} \succ \mathbf{0}$, then $\mathbf{Z} \pm \mathbf{0}$ if and only if $\Delta \mathbf{C} \pm \mathbf{0}$, where $\Delta \mathbf{C}$ is the Schur complement of $\mathbf{C}$ in $\mathbf{Z}$ and is given by $\Delta \mathbf{C}=\mathbf{A}-\mathbf{B}^{H} \mathbf{C}^{-1} \mathbf{B}$.

According to Lemma 1 and the Proposition above, the optimization problem (16) can be readily recast as an SDP:

$$
\begin{array}{lll}
\min _{\mathbf{X}, \mathbf{E}} & \operatorname{tr}(\mathbf{X}) \\
\text { s.t. } & \alpha \mathbf{I}{ }^{\circ} & \tilde{\mathbf{R}}_{\mathbf{H}_{c}}-\tilde{\mathbf{R}}_{\mathbf{H}_{c}} \mathbf{E} \tilde{\mathbf{R}}_{\mathbf{H}_{c}} \circ \beta \mathbf{I} \\
& \left\lceil\begin{array}{cc}
\mathbf{X} & \mathbf{U} \\
\mathbf{U}^{H} & \mathbf{U}^{H} \mathbf{F} \mathbf{U}
\end{array}\right] \pm \mathbf{0}
\end{array}
$$

where the matrix $\mathbf{x}$ is an auxiliary variable.

After obtaining the optimum $\mathbf{E}, \mathbf{R}_{\odot}$ can be constructed via a suitable approximation to it (in a LS sense), which is formulated as

$$
\begin{array}{ll}
\mathbf{R}_{\Phi}= & \arg \min _{\mathbf{R}_{\Phi}}\left\|\left(\mathbf{E}^{-1}-\tilde{\mathbf{R}}_{\mathbf{H}_{c}}\right)^{-1}-\tilde{\mathbf{R}}_{\Phi} \otimes \mathbf{B}^{-1}\right\|_{F} \\
\text { s.t. } & \operatorname{tr}\left(\mathbf{R}_{\Phi}\right)=L P \\
& \mathbf{R}_{\Phi} \pm \mathbf{0}
\end{array}
$$

By using the Lemma 1, similar to the discussion above, (21) can be equivalently represented as an SDP:

$$
\begin{aligned}
& \min t
\end{aligned}
$$

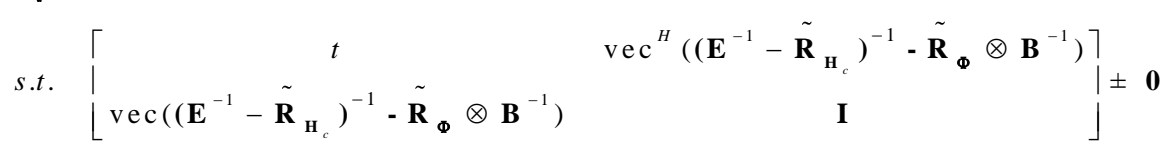

$$
\begin{aligned}
& \operatorname{tr}\left(\mathbf{R}_{\Phi}\right)=L P \\
& \mathbf{R}_{\Phi} \pm \mathbf{0}
\end{aligned}
$$

where $t$ is an auxiliary variable.

Using many well-known algorithms (see, e.g., [24]) for solving SDP problems, the problems in (20) and (22) can be solved very efficiently. In the following examples, the optimization toolbox in [25] is used for these problems. Note that we only obtain the WCM other than the ultimate transmitted waveforms in this paper. In practice, the ultimate waveforms can be asymptotically synthesized by using the method in [30]. 


\section{Numerical Examples}

In this section, we assess the effectiveness of the proposed method by using some examples, compared to the uncorrelated transmitted waveforms (i.e., $\left.\mathbf{R}_{\mathrm{s}}=\left(P / M_{t}\right) \mathbf{I}\right)$.

Consider a MIMO radar system with $M_{t}=5$ transmitting elements and $M_{r}=5$ receiving elements. The following two MIMO radar systems are exploited with various antenna configurations: MIMO radar $(0.5,0.5)$, and MIMO radar $(2.5,0.5)$, where the parameters specifying each radar system are the inter-element spacing of the transmitter and receiver (in units of wavelengths), respectively. The number of snapshots is $L=256$. The array signal-to-noise ratio (ASNR) defined as $P M_{t} M_{r} / \sigma_{\mathrm{w}}^{2}$ varies from -10 to $50 \mathrm{~dB}$ in the following examples, where $\sigma_{\mathrm{w}}^{2}$ denotes the variance of the additive white thermal noise. One target with unit amplitude at $0^{\circ}$ is considered. The clutter is modeled using discrete points, the RCSs for which are modeled as independent identical Gaussian random variables with mean zero and variance $\sigma_{i}{ }^{2}, i=1, \cdots, N_{C}$ and assumed to be fixed in the coherent processing interval (CPI). The clutter-to-noise ratio (CNR) defined as $\operatorname{tr}\left(R_{\mathrm{H}_{\mathrm{c}}}\right) / \sigma_{\mathrm{w}}^{2}$ ranges from 10 to $50 \mathrm{~dB}$. There is a strong jammer at $8^{\circ}$ with an array interference-to-noise ratio (AINR) equal to $60 \mathrm{~dB}$, defined as the product of the incident interference power and $M_{r}$ divided by $\sigma_{\mathrm{w}}^{2}$. The jammer is modeled as point source which transmits white Gaussian signal uncorrelated with the signals transmitted by MIMO radar.

Figure 1 shows the optimal transmit beampatterns with $A S N R=50 \mathrm{~dB}$ and $\mathrm{CNR}=10$ $\mathrm{dB}$. It can be seen that the proposed method places a peak at the target location, which means that the main transmitted power is focused at the target angle such that the performance of parameter estimation can be improved. Also, it is obvious that a notch is placed almost at the jammer location. Moreover, we can see grating lobes of the peak in the case of MIMO radar $(2.5,0.5)$ shown in Figure 1 (b), which is due to the sparse transmit array.
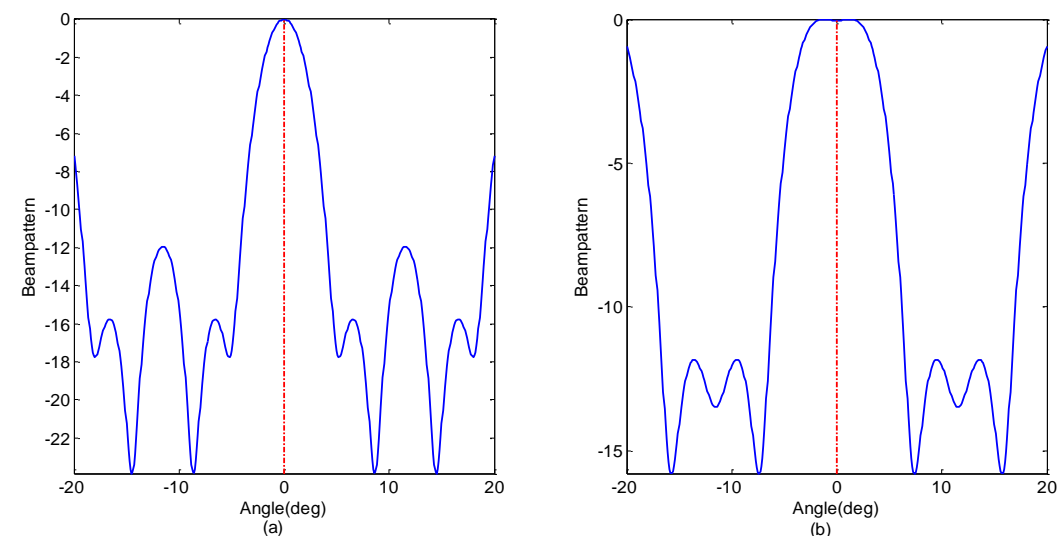

\section{Figure 1. Optimal Transmit Beampatterns with $A S N R=50 \mathrm{~dB}$ and $\mathrm{CNR}=10$ \\ dB. (a) Optimal Transmit Beampatterns for MIMO Radar $(0.5,0.5)$. (b) Optimal Transmit Beampatterns for MIMO Radar $(2.5,0.5)$}

Figure 2, shows the constrained CRB of the target angle as a function of ASNR or CNR. One can see that the CRB obtained by the proposed method or uncorrelated waveforms decreases as the increasing of ASNR, while increases as the decreasing of CNR. Moreover, the CRB obtained by the proposed method is much lower than that of uncorrelated waveforms, regardless of ASNR or CNR. Furthermore, it can be seen from Figure 2 (a), or (b), that the total CRB for MIMO radar $(2.5,0.5)$ is lower than that for 
MIMO radar $(0.5,0.5)$, which is due to the fact that the virtual receiving array aperture for the former radar is much larger than that for the latter [1].
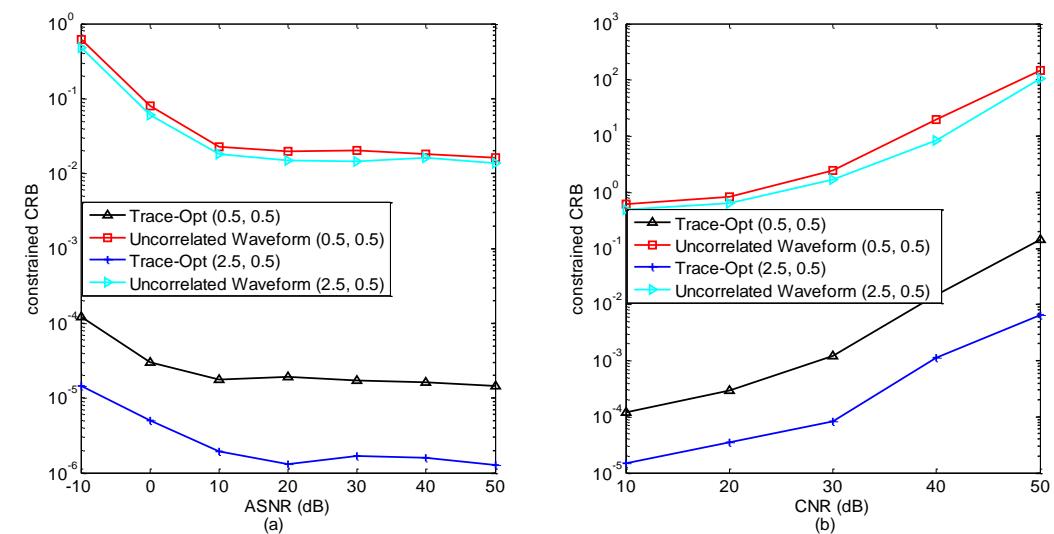

Figure 2. Constrained CRB Obtained by the Proposed Method, Along with That of the Uncorrelated Waveforms, Versus ASNR or CNR. (a) Constrained CRB Versus ASNR with $C N R=10 \mathrm{~dB}$. (b) Constrained CRB Versus CNR with ASNR=-10 dB

In order to examine the effect of the prior information on the CRB, Figure 3 , shows the $\mathrm{CRB}$ with and without the prior information as well as that obtained by uncorrelated waveforms as a function of ASNR or CNR. One can observe that the optimized transmitted waveforms with the prior information can significantly improve the accuracy of parameter estimation for each MIMO radar configuration, as compared to that without the prior information and uncorrelated waveforms, regardless of ASNR or CNR.
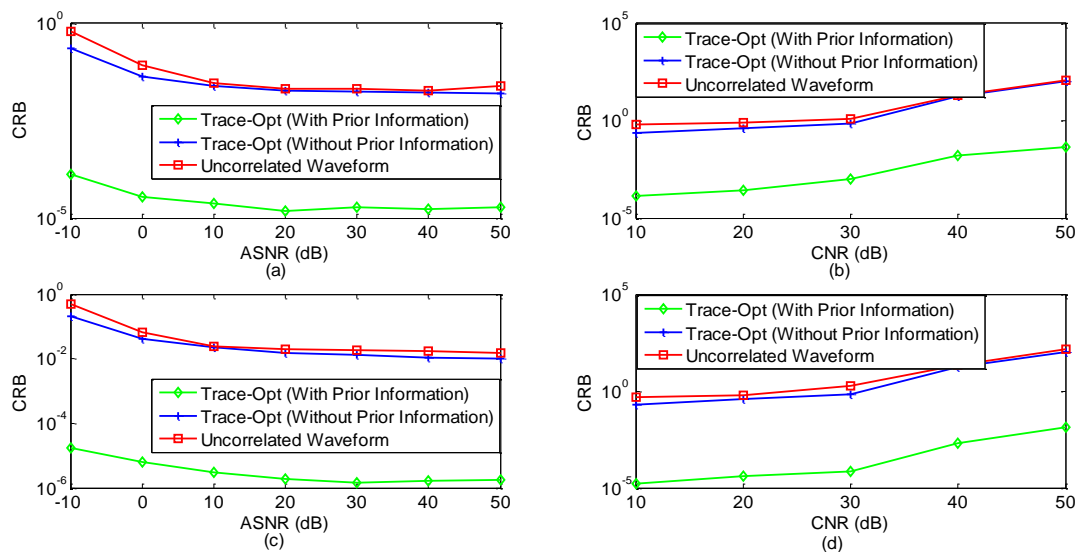

Figure 3. CRB Obtained Only by Using the Prior Information, Along with That of That without the Prior ilformation and the Uncorrelated Waveforms, Versus ASNR or CNR. (a) CRB Versus ASNR with CNR $=10 \mathrm{~dB}$ for MIMO Radar $(0.5,0.5)$. (b) CRB Versus CNR with ASNR=-10 dB for MIMO Radar $(0.5,0.5)$. (c) CRB Versus ASNR with CNR=10 dB for MIMO Radar $(2.5,0.5)$. (d) CRB Versus CNR with ASNR=-10 dB for MIMO Radar $(2.5,0.5)$

\section{Conclusions}

In this paper, we have investigated the problem of waveform optimization with the prior information of targets of interests to improve the parameter estimation performance of MIMO radar in the presence of clutter, which is based on the 
constrained $\mathrm{CRB}$. In order to solve the resultant nonlinear optimization problem, a novel DL based algorithm has been proposed to relax this problem as an SDP issue, which can be solved very efficiently. Following that, an optimal solution to the initial problem has been obtained via the LS fitting of the solution of the SDP one. Numerical examples show that the optimized transmitted waveforms generated by the proposed method can significantly improve the accuracy of parameter estimation, as compared to the optimized transmitted waveforms without prior information and uncorrelated waveforms.

\section{Appendix A}

\section{Fisher Information Matrix}

According to [14], the constrained CRB can be written as Based on the signal model in (3), and stack the columns of $\mathbf{Y}$ in a $M_{r} L \times 1$ vector as

$$
\begin{aligned}
\operatorname{vec}(\mathbf{Y}) & =\left(\boldsymbol{\Phi}^{T} \otimes \mathbf{I}_{M_{r}}\right) \sum_{k=1}^{K} \beta_{k}\left(\mathbf{v}\left(\theta_{k}\right) \otimes \mathbf{a}\left(\theta_{k}\right)\right)+\left(\boldsymbol{\Phi}^{T} \otimes \mathbf{I}_{M_{r}}\right) \operatorname{vec}\left(\mathbf{H}_{c}\right)+\operatorname{vec}(\mathbf{W}) \\
& =\left(\boldsymbol{\Phi}^{T} \otimes \mathbf{I}_{M_{r}}\right)(\mathbf{V} * \mathbf{A}) \boldsymbol{\beta}+\left(\boldsymbol{\Phi}^{T} \otimes \mathbf{I}_{M_{r}}\right) \operatorname{vec}\left(\mathbf{H}_{c}\right)+\operatorname{vec}(\mathbf{W})
\end{aligned}
$$

In this paper, we calculate the FIM with respect to $\boldsymbol{\theta}, \boldsymbol{\beta}_{R}, \boldsymbol{\beta}_{I}$ (Here we only consider one-dimensional targets.). According to [28], we have

$$
F\left(\theta_{i}, \theta_{j}\right)=2 \operatorname{Re}\left[\operatorname{tr}\left(\frac{\partial\left(\left(\boldsymbol{\Phi}^{T} \otimes \mathbf{I}_{M_{r}}\right)(\mathbf{V} * \mathbf{A}) \boldsymbol{\beta}\right)^{H}}{\partial \theta_{i}} \mathbf{Q}^{-1} \frac{\partial\left(\left(\boldsymbol{\Phi}^{T} \otimes \mathbf{I}_{M_{r}}\right)(\mathbf{V} * \mathbf{A}) \boldsymbol{\beta}\right)}{\partial \theta_{i}}\right)\right]
$$

where $\mathbf{Q}$ denotes the covariance of the clutter plus interference and noise, which can be represented as

$\mathbf{Q}=E\left\{\left[\left(\boldsymbol{\Phi}^{T} \otimes \mathbf{I}_{M_{r}}\right) \operatorname{vec}\left(\mathbf{H}_{c}\right)+\operatorname{vec}(\mathbf{W})\right]\left[\left(\boldsymbol{\Phi}^{T} \otimes \mathbf{I}_{M_{r}}\right) \operatorname{vec}\left(\mathbf{H}_{c}\right)+\operatorname{vec}(\mathbf{W})\right]^{H}\right\}$

With (4), the equation above can be simplified as

$\mathbf{Q}=\left(\Phi^{T} \otimes \mathbf{I}_{M_{r}}\right) \mathbf{R}_{\mathbf{H}_{c}}\left(\boldsymbol{\Phi}^{*} \otimes \mathbf{I}_{M_{r}}\right)+\mathbf{I}_{M_{t}} \otimes \mathbf{B}$

Because

$$
\begin{aligned}
\frac{\partial\left(\left(\boldsymbol{\Phi}^{T} \otimes \mathbf{I}_{M_{r}}\right)(\mathbf{V} * \mathbf{A}) \boldsymbol{\beta}\right)}{\partial \theta_{i}}= & \left(\boldsymbol{\Phi}^{T} \otimes \mathbf{I}_{M_{r}}\right)\left(\left(\dot{\mathbf{V}} \mathbf{e}_{i} \mathbf{e}_{i}^{T} * \mathbf{A}\right) \boldsymbol{\beta}+\left(\mathbf{V} * \dot{\mathbf{A}} \mathbf{e}_{i} \mathbf{e}_{i}^{T}\right) \boldsymbol{\beta}\right) \\
& =\left(\boldsymbol{\Phi}^{T} \otimes \mathbf{I}_{M_{r}}\right)\left((\dot{\mathbf{V}} * \mathbf{A}) \mathbf{e}_{i} \mathbf{e}_{i}^{T} \boldsymbol{\beta}+(\mathbf{V} * \dot{\mathbf{A}}) \mathbf{e}_{i} \mathbf{e}_{i}^{T} \boldsymbol{\beta}\right)
\end{aligned}
$$

where $\mathbf{e}_{i}$ represents the ith column of the identity matrix, then

$$
\begin{aligned}
F\left(\theta_{i}, \theta_{j}\right)= & 2 \operatorname{Re}\left\{\operatorname { t r } \left[\left[\left(\boldsymbol{\Phi}^{T} \otimes \mathbf{I}_{M_{r}}\right)\left((\dot{\mathbf{V}} * \mathbf{A}) \mathbf{e}_{i} \mathbf{e}_{i}^{T} \boldsymbol{\beta}+(\mathbf{V} * \dot{\mathbf{A}}) \mathbf{e}_{i} \mathbf{e}_{i}^{T} \boldsymbol{\beta}\right)\right]^{H}\right.\right. \\
& \left.\left.\times \mathbf{Q}^{-1}\left[\left(\boldsymbol{\Phi}^{T} \otimes \mathbf{I}_{M_{r}}\right)\left((\dot{\mathbf{V}} * \mathbf{A}) \mathbf{e}_{j} \mathbf{e}_{j}^{T} \boldsymbol{\beta}+(\mathbf{V} * \dot{\mathbf{A}}) \mathbf{e}_{j} \mathbf{e}_{j}^{T} \boldsymbol{\beta}\right)\right]\right]\right\}
\end{aligned}
$$

Besides, note that 


$$
\begin{aligned}
& \operatorname{tr}\left\{\left[\left(\boldsymbol{\Phi}^{T} \otimes \mathbf{I}_{M_{r}}\right)\left((\dot{\mathbf{V}} * \mathbf{A}) \mathbf{e}_{i} \mathbf{e}_{i}^{T} \boldsymbol{\beta}\right]^{H} \mathbf{Q}^{-1}\left[\left(\boldsymbol{\Phi}^{T} \otimes \mathbf{I}_{M_{r}}\right)\left((\dot{\mathbf{V}} * \mathbf{A}) \mathbf{e}_{j} \mathbf{e}_{j}^{T} \boldsymbol{\beta}\right]\right\}\right.\right. \\
& =\operatorname{tr}\left\{\boldsymbol{\beta}^{H} \mathbf{e}_{i} \mathbf{e}_{i}^{T}(\dot{\mathbf{V}} * \mathbf{A})^{H}\left(\boldsymbol{\Phi}^{*} \otimes \mathbf{I}_{M_{r}}\right) \mathbf{Q}^{-1}\left(\boldsymbol{\Phi}^{T} \otimes \mathbf{I}_{M_{r}}\right)\left((\dot{\mathbf{V}} * \mathbf{A}) \mathbf{e}_{j} \mathbf{e}_{j}^{T} \boldsymbol{\beta}\right\}\right.
\end{aligned}
$$

Let $\mathbf{T}=\left(\boldsymbol{\Phi}^{*} \otimes \mathbf{I}_{M_{r}}\right)\left[\left(\boldsymbol{\Phi}^{T} \otimes \mathbf{I}_{M_{r}}\right) \mathbf{R}_{\mathbf{H}_{c}}\left(\boldsymbol{\Phi}^{*} \otimes \mathbf{I}_{M_{r}}\right)+\mathbf{I}_{M_{q}} \otimes \mathbf{B}\right]^{-1}\left(\boldsymbol{\Phi}^{T} \otimes \mathbf{I}_{M_{r}}\right)$. By using matrix inversion lemma, we can get

$$
\begin{aligned}
\mathbf{T} & =\left(\boldsymbol{\Phi}^{*} \otimes \mathbf{I}_{M_{r}}\right)\left\{\mathbf{I}_{M_{c}} \otimes \mathbf{B}^{-1}-\left(\boldsymbol{\Phi}^{T} \otimes \mathbf{B}^{-1}\right) \mathbf{R}_{\mathbf{H}_{c}}\left[\mathbf{I}_{M_{t} M_{r}}+\left(\left(\boldsymbol{\Phi}^{*} \boldsymbol{\Phi}^{T}\right) \otimes \mathbf{B}^{-1}\right) \mathbf{R}_{\mathbf{H}_{c}}\right]^{-1}\left(\boldsymbol{\Phi}^{*} \otimes \mathbf{B}^{-1}\right)\right\}\left(\boldsymbol{\Phi}^{T} \otimes \mathbf{I}_{M_{r}}\right) \\
& =\left(\boldsymbol{\Phi}^{*} \boldsymbol{\Phi}^{T}\right) \otimes \mathbf{B}^{-1}-\left(\left(\boldsymbol{\Phi}^{*} \boldsymbol{\Phi}^{T}\right) \otimes \mathbf{B}^{-1}\right) \mathbf{R}_{\mathbf{H}_{c}}\left[\mathbf{I}_{M_{c_{r}} M_{r}}+\left(\left(\boldsymbol{\Phi}^{*} \boldsymbol{\Phi}^{T}\right) \otimes \mathbf{B}^{-1}\right) \mathbf{R}_{\mathbf{H}_{c}}\right]^{-1}\left(\boldsymbol{\Phi}^{*} \boldsymbol{\Phi}^{T}\right) \otimes \mathbf{B}^{-1} \\
& =\left(\mathbf{I}_{M_{c} M_{r}}+\left(\mathbf{R}_{\boldsymbol{\Phi}} \otimes \mathbf{B}^{-1}\right) \mathbf{R}_{\mathbf{H}_{c}}\right)^{-1}\left(\mathbf{R}_{\boldsymbol{\Phi}} \otimes \mathbf{B}^{-1}\right)
\end{aligned}
$$

where $\mathbf{R}_{\Phi}=\Phi^{*} \Phi^{T}$.

Based the discussion above, we can get that

$$
\begin{aligned}
\operatorname{tr}\left\{\boldsymbol{\beta}^{H} \mathbf{e}_{i} \mathbf{e}_{i}^{T}(\dot{\mathbf{V}} * \mathbf{A})^{H} \mathbf{T}\left((\dot{\mathbf{V}} * \mathbf{A}) \mathbf{e}_{j} \mathbf{e}_{j}^{T} \boldsymbol{\beta}\right\}\right. & =\operatorname{tr}\left\{\mathbf{e}_{i}^{T}(\dot{\mathbf{V}} * \mathbf{A})^{H} \mathbf{T}\left((\dot{\mathbf{V}} * \mathbf{A}) \mathbf{e}_{j} \mathbf{e}_{j}^{T} \boldsymbol{\beta} \boldsymbol{\beta}^{H} \mathbf{e}_{i}\right\}\right. \\
& =\mathbf{e}_{i}^{T}(\dot{\mathbf{V}} * \mathbf{A})^{H} \mathbf{T}\left((\dot{\mathbf{V}} * \mathbf{A}) \mathbf{e}_{j} \mathbf{e}_{j}^{T} \boldsymbol{\beta} \boldsymbol{\beta}^{H} \mathbf{e}_{i}\right. \\
& =\left((\dot{\mathbf{V}} * \mathbf{A})^{H} \mathbf{T}((\dot{\mathbf{V}} * \mathbf{A}))_{i j}\left(\boldsymbol{\beta}^{*} \boldsymbol{\beta}^{T}\right)_{i j}\right.
\end{aligned}
$$

where $\mathbf{X}_{i j}$ denotes the $(\mathrm{i}, \mathrm{j})$ th element of $\mathbf{X}$. Hence,

$F\left(\theta_{i}, \theta_{j}\right)=2 \operatorname{Re}\left[\mathbf{F}_{11}\right]_{i j}, \quad$ i.e., $\quad F(\boldsymbol{\theta}, \boldsymbol{\theta})=2 \operatorname{Re}\left[\mathbf{F}_{11}\right]$

with $\mathbf{F}_{11}$ given in (10).

Similarly, we have

$\frac{\partial\left(\left(\boldsymbol{\Phi}^{T} \otimes \mathbf{I}_{M_{r}}\right)(\mathbf{V} * \mathbf{A}) \boldsymbol{\beta}\right)}{\partial \beta_{R, i}}=\left(\Phi^{T} \otimes \mathbf{I}_{M_{r}}\right)(\mathbf{V} * \mathbf{A}) \mathbf{e}_{i}$

and

$\frac{\partial\left(\left(\boldsymbol{\Phi}^{T} \otimes \mathbf{I}_{M_{r}}\right)(\mathbf{V} * \mathbf{A}) \boldsymbol{\beta}\right)}{\partial \beta_{I, i}}=j\left(\boldsymbol{\Phi}^{T} \otimes \mathbf{I}_{M_{r}}\right)(\mathbf{V} * \mathbf{A}) \mathbf{e}_{i}$

Hence

$\mathbf{F}\left(\boldsymbol{\theta}, \boldsymbol{\beta}_{R}\right)=\mathbf{F}^{T}\left(\boldsymbol{\theta}, \boldsymbol{\beta}_{R}\right)=2 \operatorname{Re}\left(\mathbf{F}_{12}\right)$

and

$\mathbf{F}\left(\boldsymbol{\theta}, \boldsymbol{\beta}_{I}\right)=\mathbf{F}^{T}\left(\boldsymbol{\theta}, \boldsymbol{\beta}_{I}\right)=-2 \operatorname{Im}\left(\mathbf{F}_{12}\right)$

where $\mathbf{F}_{12}$ is given in (11).

We also have

$\mathbf{F}\left(\boldsymbol{\beta}_{R}, \boldsymbol{\beta}_{R}\right)=\mathbf{F}\left(\boldsymbol{\beta}_{I}, \boldsymbol{\beta}_{I}\right)=2 \operatorname{Re}\left(\mathbf{F}_{22}\right)$

and

$\mathbf{F}\left(\boldsymbol{\beta}_{I}, \boldsymbol{\beta}_{R}\right)=\mathbf{F}^{T}\left(\boldsymbol{\beta}_{R}, \boldsymbol{\beta}_{I}\right)=-2 \operatorname{Im}\left(\mathbf{F}_{22}\right)$ 
where $\mathbf{F}_{22}$ is given in (12).

From (32) and (35)-(38), we can obtain (9) immediately.

\section{Appendix B}

\section{Proof of Proposition}

In order to reshape the objective in (16) as a linear function, let

$\mathbf{E}=\left(\mathbf{I}+\left(\tilde{\mathbf{R}}_{\Phi} \otimes \mathbf{B}^{-1}\right) \tilde{\mathbf{R}}_{\mathbf{H}_{c}}\right)^{-1}\left(\tilde{\mathbf{R}}_{\Phi} \otimes \mathbf{B}^{-1}\right)$

Substitute (39) into the objective in (16), we can see that the outer optimization is the linear function with respect to $\mathbf{E}$.

Let $\mathbf{M}=\left(\tilde{\mathbf{R}}_{\Phi} \otimes \mathbf{B}^{-1}\right) \tilde{\mathbf{R}}_{\mathbf{H}_{c}}$, because

$$
(\mathbf{I}+\mathbf{M})^{-1}(\mathbf{I}+\mathbf{M})=\mathbf{I}
$$

then,

$(\mathbf{I}+\mathbf{M})^{-1} \mathbf{M}=\mathbf{I}-(\mathbf{I}+\mathbf{M})^{-1}$

and

$\mathbf{I}-(\mathbf{I}+\mathbf{M})^{-1}=\mathbf{E} \tilde{\mathbf{R}}_{\mathbf{H}_{c}}$

Hence,

$\left(\left(\mathbf{I}-\mathbf{E} \tilde{\mathbf{R}}_{\mathbf{H}_{c}}\right)^{-1}-\mathbf{I}\right) \tilde{\mathbf{R}}_{\mathbf{H}_{c}}^{-1}=\tilde{\mathbf{R}}_{\Phi} \otimes \mathbf{B}^{-1}$

Following it, we can obtain,

$\left(\tilde{\mathbf{R}}_{\mathbf{H}_{c}}-\tilde{\mathbf{R}}_{\mathbf{H}_{c}} \mathbf{E} \tilde{\mathbf{R}}_{\mathbf{H}_{c}}\right)^{-1}=\tilde{\mathbf{R}}_{\Phi} \otimes \mathbf{B}^{-1}+\tilde{\mathbf{R}}_{\mathbf{H}_{c}}^{-1}$

According to Weyl's theorem [29], we can obtain

$$
\begin{aligned}
& \lambda_{\max }\left(\left(\tilde{\mathbf{R}}_{\mathbf{H}_{c}}-\tilde{\mathbf{R}}_{\mathbf{H}_{c}} \mathbf{E} \tilde{\mathbf{R}}_{\mathbf{H}_{c}}\right)^{-1}\right) \leq \lambda_{\max }\left(\tilde{\mathbf{R}}_{\Phi} \otimes \mathbf{B}^{-1}\right)+\lambda_{\max }\left(\tilde{\mathbf{R}}_{\mathbf{H}_{c}}^{-1}\right) \\
& \lambda_{\text {min }}\left(\left(\tilde{\mathbf{R}}_{\mathbf{H}_{c}}-\tilde{\mathbf{R}}_{\mathbf{H}_{c}} \mathbf{E} \tilde{\mathbf{R}}_{\mathbf{H}_{c}}\right)^{-1}\right) \geq \lambda_{\min }\left(\tilde{\mathbf{R}}_{\Phi} \otimes \mathbf{B}^{-1}\right)+\lambda_{\text {min }}\left(\tilde{\mathbf{R}}_{\mathbf{H}_{c}}^{-1}\right)
\end{aligned}
$$

where $\lambda_{\min }(\cdot)$ denotes the smallest eigenvalue of a matrix. Based on the fact that

$\lambda_{\max }\left(\tilde{\mathbf{R}}_{\Phi} \otimes \mathbf{B}^{-1}\right) \leq \lambda_{\max }\left(\tilde{\mathbf{R}}_{\Phi}\right) \lambda_{\max }\left(\mathbf{B}^{-1}\right)$

$\lambda_{\text {min }}\left(\tilde{\mathbf{R}}_{\Phi} \otimes \mathbf{B}^{-1}\right) \geq \lambda_{\min }\left(\tilde{\mathbf{R}}_{\Phi}\right) \lambda_{\min }\left(\mathbf{B}^{-1}\right)$

(45) can be equivalently represented as

$\lambda_{\text {max }}\left(\left(\tilde{\mathbf{R}}_{\mathbf{H}_{c}}-\tilde{\mathbf{R}}_{\mathbf{H}_{c}} \mathbf{E} \tilde{\mathbf{R}}_{\mathbf{H}_{c}}\right)^{-1}\right) \leq \lambda_{\max }\left(\tilde{\mathbf{R}}_{\boldsymbol{\Phi}}\right) \lambda_{\max }\left(\mathbf{B}^{-1}\right)+\lambda_{\max }\left(\tilde{\mathbf{R}}_{\mathbf{H}_{c}}^{-1}\right)$

$\lambda_{\text {min }}\left(\left(\tilde{\mathbf{R}}_{\mathbf{H}_{c}}-\tilde{\mathbf{R}}_{\mathbf{H}_{c}} \mathbf{E} \tilde{\mathbf{R}}_{\mathbf{H}_{c}}\right)^{-1}\right) \geq \lambda_{\text {min }}\left(\tilde{\mathbf{R}}_{\Phi}\right) \lambda_{\text {min }}\left(\mathbf{B}^{-1}\right)+\lambda_{\text {min }}\left(\tilde{\mathbf{R}}_{\mathbf{H}_{c}}^{-1}\right)$

Note that

$\lambda_{\text {max }}\left(\mathbf{B}^{-1}\right)=\lambda_{\text {min }}(\mathbf{B}), \lambda_{\text {min }}\left(\mathbf{B}^{-1}\right)=\lambda_{\text {max }}(\mathbf{B})$

$\lambda_{\text {max }}\left(\tilde{\mathbf{R}}_{\mathbf{H}_{c}}^{-1}\right)=\lambda_{\text {min }}\left(\tilde{\mathbf{R}}_{\mathbf{H}_{c}}\right), \lambda_{\text {min }}\left(\tilde{\mathbf{R}}_{\mathbf{H}_{c}}^{-1}\right)=\lambda_{\text {max }}\left(\tilde{\mathbf{R}}_{\mathbf{H}_{c}}\right)$

With (17) and (48), (47) can be recast as 


$$
\begin{aligned}
& \lambda_{\text {max }}\left(\left(\tilde{\mathbf{R}}_{\mathbf{H}_{c}}-\tilde{\mathbf{R}}_{\mathbf{H}_{c}} \mathbf{E} \tilde{\mathbf{R}}_{\mathbf{H}_{c}}\right)^{-1}\right) \leq(L P+\varepsilon) \lambda_{\text {min }}(\mathbf{B})+\mu+\lambda_{\text {min }}\left(\mathbf{R}_{\mathbf{H}_{c}}\right) \\
& \lambda_{\text {min }}\left(\left(\tilde{\mathbf{R}}_{\mathbf{H}_{c}}-\tilde{\mathbf{R}}_{\mathbf{H}_{c}} \mathbf{E} \tilde{\mathbf{R}}_{\mathbf{H}_{c}}\right)^{-1}\right) \geq \varepsilon \lambda_{\text {max }}(\mathbf{B})+\mu+\lambda_{\text {max }}\left(\mathbf{R}_{\mathbf{H}_{c}}\right)
\end{aligned}
$$

As a sequence, we can obtain

$$
\alpha \mathbf{I} \circ \tilde{\mathbf{R}}_{\mathbf{H}_{c}}-\tilde{\mathbf{R}}_{\mathbf{H}_{c}} \mathbf{E} \tilde{\mathbf{R}}_{\mathbf{H}_{c}}{ }^{\circ} \beta \mathbf{I}
$$

where

$$
\alpha=\frac{1}{(L P+\varepsilon) \lambda_{\text {min }}(\mathbf{B})+\mu+\lambda_{\text {min }}\left(\mathbf{R}_{\mathbf{H}_{c}}\right)}
$$

and

$$
\beta=\frac{1}{\varepsilon \lambda_{\text {max }}(\mathbf{B})+\mu+\lambda_{\text {max }}\left(\mathbf{R}_{\mathbf{H}_{c}}\right)}
$$

With (50)-(52), (18) holds immediately.

\section{Acknowledgments}

The author would like to thank Dr. Hongyan Wang, and the anonymous reviewers for their thoughtful and to-the-point comments and suggestions, which greatly improved the manuscript. This work is sponsored in part by NSFC under Grant 61301258, the development project of Henan Provincial Department of science and technology under Grant 142300410402, the Education Department of Henan province university innovation talent support program under Grant 2012HASTIT032, and the scientific research fund of Henan Provincial Education Department under Grant 14B520057.

\section{References}

[1] J. Li and P. Stoica, "MIMO radar with colocated antennas", IEEE Signal Processing Magazine, vol. 24, no. 5 , (2007), pp. 106-114.

[2] E. Fishler, A. Haimovich, R. Blum, L. Cimini, D. Chizhik and R. Valenzuela, "Spatial diversity in radars-models and detection performance", IEEE Trans. on Signal Processing, vol. 54, no. 3, (2006), pp. 823-838.

[3] J. Li, P. Stoica, L. Xu and W. Roberts, "On parameter identifiability of MIMO radar", IEEE Trans. on Signal Processing Lett., vol. 14, no. 12, (2007), pp. 968-971.

[4] H. Wang, G. Liao, Y. Wang and X. Liu, "On parameter identifiability of MIMO radar with waveform diversity”, Signal Processing, vol. 91, no. 8, (2011), pp. 2057-2063.

[5] S. Peter, J. Li and Y. Xie, "On Probing Signal Design For MIMO Radar", IEEE Trans. on Signal Processing, vol. 55, no. 8, (2007), pp. 4151-4161.

[6] D. R. Fuhrmann and G. S. Antonio, "Transmit beamforming for MIMO radar systems using signal cross-correlation", IEEE Trans. on Aerospace and Electronic System, vol. 44, no. 1, (2008), pp. 171186.

[7] G. Cui, H. Li and M. Rangaswamy, "MIMO Radar Waveform Design With Constant Modulus and Similarity Constraints", IEEE Trans. on Signal Processing, vol. 62, no. 2, (2014), pp. 343-353.

[8] Y. C. Wang, X. Wang, H. Liu and Z. Luo, "On the Design of Constant Modulus Probing Signals for MIMO Radar", IEEE Trans. on Signal Processing, vol. 60, no. 8, (2012), pp. 4432-4438.

[9] A. D. Maio, S. D. Nicola, Y. Huang, S. Zhang and A. Farina, "Code design to optimize radar detection performance under accuracy and similarity constraints", IEEE Trans. on Signal Processing, vol. 56, no. 11, (2008), pp. 5618-5629.

[10] E. Grossi, M. Lops and L. Venturino, "Robust Waveform Design for MIMO Radars", IEEE Trans. on Signal Processing, vol. 59, no. 7, (2011), pp. 3262-3271.

[11] C. Y. Chen and P. P. Vaidyanathan, "MIMO Radar Ambiguity Properties and Optimization Using Frequency-Hopping Waveforms", IEEE Trans. on Signal Processing, vol. 56, no.12, (2008), pp. 59265936.

[12] J. Li, L. Xu, P. Stoica, K. W. Forsythe and D. W. Bliss, "Range Compression and Waveform Optimization for MIMO Radar: A Cramer-Rao Bound Based Study", IEEE Trans. on Signal Processing, vol. 56, no. 1, (2008), pp. 218-232. 
[13] B. Friedlander, "Waveform Design for MIMO Radars", IEEE Trans. on Aerospace and Electronic Systems, vol. 43, no. 3, (2007), pp. 1227-1238.

[14] C. Y. Chen and P. P. Vaidyanathan, "MIMO Radar Waveform Optimization with Prior Information of the Extended Target and Clutter", IEEE Trans. on Signal Processing, vol. 57, no. 9, (2009), pp. 35333544.

[15] Y. Yang and R. Blum, "MIMO Radar Waveform Design Based on Mutual Information and Minimum Mean-Square Error Estimation", IEEE Trans. on Aerospace and Electronic Systems, vol. 43, no. 1, (2007), pp. 330-343.

[16] Y. Yang and R. Blum, "Minimax Robust MIMO Radar Waveform Design", IEEE Journal of Selected Topics in Signal Processing, vol. 1, no. 1, (2007), pp. 147-155.

[17] B. Tang, J. Tang and Y. Pen, "Waveform Optimization for MIMO Radar in Colored Noise: Further Results for Estimation-Oriented Criteria”, IEEE Trans. on Signal Processing, vol. 60, no. 3, (2012), pp. $1517-1522$.

[18] A. Leshem, O. Naparstek and A. Nehorai, "Information Theoretic Adaptive Radar Waveform Design for Multiple Extended Targets", IEEE Journal of Selected Topics in Signal Processing, vol. 1, no. 1, (2007), pp. 42-55.

[19] T. Naghibi, M. Namvar and F. Behnia, "Optimal and robust waveform design for MIMO radars in the presence of clutter", Signal Processing, vol. 90, no. 4, (2010), pp. 1103-1117.

[20] J. Liu, H. Li and B. Himed, "Joint Optimization of Transmit and Receive Beamforming in Active Arrays”, IEEE Trans. on Signal Processing Lett., vol. 21, no. 1, (2014), pp. 39-42.

[21] M. B. Sadler, J. R. Kozick and T.Moore, "Bounds on Bearing and Symbol Estimation with Side Information”, IEEE Trans. Signal Processing, vol. 49, no. 4, (2001), pp. 822-834.

[22] J. D. Gorman and A. O. Hero, "Lower bounds for parametric estimation with constraints", IEEE Trans. Information Theory, vol. 26, no. 6, (1990), pp. 1285-1301.

[23] H. Cox, R. M. Zeskind and M. H. Owen, "Robust adaptive beam-forming", IEEE Trans. Acoust., Speech, Signal Processing, vol. 35, (1985), pp. 1365-1376.

[24] A. B. Tal and A. Nemirovski, "Lectures on Modern Convex Optimization", ser. Optimization. Philadelphia, PA: MPS-SIAM, (2001).

[25] J. Lofberg, "YALMIP: A Toolbox for Modeling and Optimization in MATLAB", In Proceedings of the CACSD Conference, Taipei, Taiwan, (2004), pp. 284-289.

[26] C. Y. Chen and P. P. Vaidyanathan, "MIMO Radar Space-Time Adaptive Processing Using Prolate Spheroidal Wave Functions", IEEE Trans. on Signal Processing, vol. 56, no. 2, (2008), pp. 623-635.

[27] L. Xu, J. Li and P. Stoica, "Target Detection and Parameter Estimation for MIMO Radar Systems", IEEE Trans. Aerospace and Electronic System, vol. 44, no. 3, (2008), pp. 927-939.

[28] P. Stoica and R. L. Moses, "Spectral Analysis of Signals", NJ: Prentice-Hall, Upper SaddleRiver, (2005).

[29] R. A. Horn and C. R. Johnson, "Matrix Analysis", Cambridge Univ. Press, Cambridge, U.K., (1985).

[30] P. Stoica, J. Li and X. Zhu, "Waveform Synthesis for Diversity-Based Transmit Beampattern Design", IEEE Trans. On Signal Processing, vol. 56, no. 1, (2008), pp. 2593-2598.

\section{Author}

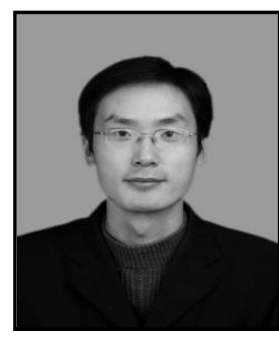

Hongfeng Wang, he received the M.E. degree in computer application technology from HuaZhong University of Science and Technology of China in 2012. He joined School of Computer Science and Technology, Zhoukou Normal University in 2004, where he is currently lecture. His research interests are mainly in space-time adaptive processing, signal processing for radar. 
International Journal of Signal Processing, Image Processing and Pattern Recognition Vol. 9, No. 8 (2016) 